\title{
Features of the New Defect Indicator on the Gorbunov Effect
}

\author{
Sutorikhin Vladimir \\ Faculty of Radio Engineering, Tomsk University of Control Systems and Electronics, Tomsk, Russia \\ Email address: \\ winddiad1@yandex.ru

\section{To cite this article:} \\ Sutorikhin Vladimir. Features of the New Defect Indicator on the Gorbunov Effect. American Journal of Aerospace Engineering. \\ Vol. 6, No. 1, 2019, pp. 24-26. doi: 10.11648/j.ajae.20190601.14
}

Received: July 21, 2019; Accepted: August 21, 2019; Published: September 4, 2019

\begin{abstract}
It's been 20 years since the opening of the new phenomenon of ultrasonic treatment on defects in the metal, which ingibiruetsa Doppler radar. The former results from the application of radar to monitor defects limited to surface discontinuities under the paint layer, other dielectric coatings. A new direction of remote detection of metal defects (cracks, metal stratification at a depth of several tens of centimeters) by Doppler radar of 30-40GHz frequency is now possible. Developed, tested sensor contactless indexing of acts of acoustic emission. A device for indicating defects that could previously be detected only by contact sensors based on piezoelectrics was tested. The paper deals with the causes of previous failures, low sensitivity of radars with their direct $p$. the possibilities of increasing the sensitivity due to the joint use of ultrasound and microwave radiation are Shown. The obtained results require a revision of the capabilities of the known ultrasonic flaw detectors of active and passive principle of action.
\end{abstract}

Keywords: Active, Passive Ultrasonic Methods, Gorbunov Effect, Doppler Radar

\section{Introduction}

In the middle of the last century, the problem of replacing contact sensors used in ultrasonic testing of metals with remote sensors comparable in their action to Doppler radars became urgent. The practice of using contact sensors is faced with a high complexity of diagnostics of moving objects [1]. These include railway wheels, generator shafts and turbines of thermal power plants. There were difficulties in determining the defects of special products (zirconium tubes for Heat Emitting Elements of nuclear power plants). Restrictions on the use of contact ultrasonic sensors also applied to surfaces heated above $300^{\circ}$, at which there is a loss of sensor sensitivity. In addition, it soon became clear that the dynamic range of sensitive sensors from piezoceramics (45-50 dB), much lower than the dynamic range of the studied signals $(90-120 \mathrm{~dB})$ [2]. For this reason, often occur the destruction of sensors, loss of sensitivity during the critical trials. It was assumed that the interaction of the microwave field with the metal surface, with active and passive ultrasound diagnosis can be easily detected. Moreover, the excitation of the metal surface by laser excitation of ultrasonic waves was no longer a difficult task
[3]. In the period 1970-1980 American ultrasound generators were produced by the military industry. For this reason, it was believed that the replacement of contact sensors with laser or microwave in the near future. But it soon became clear that the sensitive laser sensor surface deformation was very weak. A useful signal from ultrasound sources could be detected on the surface with a laser only at amplitude of a few microns, while contact sensors could detect the vibration amplitudes of a unit of nanometer. Later, this trouble has not been overcome radically. Laser sensors could detect "movements" of a metal surface with a value of 0.1-0.5 nanometers, but these movements were applied only to artificial simulators, which are mirror-treated surfaces of very small size (10-20microns) [4]. The practice of using laser "Speckle" conventional surfaces faced with a high level of complex processing, which does not allow investigating surface vibrations with a frequency of more than 100 Hertz.

As usual, the first complex studies in the open literature there are many patents describing the possible implementation of microwave sensors, replacing the contact acoustic emission [5-8], however, the practical test has not confirmed the "light of hope". The useful signal at the output of Doppler radars of different frequencies (from 10 to 
$40 \mathrm{GHz}$ ) was below the noise level even for temperatures close to absolute zero. An example is the publication of the American researcher Gregory Smith [4], who not only describes the possibility of implementing his invention, which completely repeats the Russian copyright certificate published twenty years earlier [5], but also gives the results contradicting the known measurements of the parameters of acoustic emission of metals. However, both the first and the second invention proved to be untenable in practice. Nature does not allow direct registration of vibrations of the metal surface with an amplitude of less than a hundred nanometers. Within the limits as long as the linear Hooke law is satisfied.

\section{Researches}

Complex, long-term work of Russian researchers under the guidance of Professor V. I. Gorbunov showed that direct measurement of acoustic emission parameters by Doppler radar is impossible. The reason is that the level of phase modulation of the microwave field reflected from the surface is close to zero [9]. Surface changes of an elastic wave consist of two components. The first it is self-oscillation the surface. The second is dependence of the metal density in this wave. The higher the oscillation amplitude, the lower the metal density in the amplitude. Since the density of the metal is directly proportional to the density of the conductivity particles (electrons), it turns out that changes in the phase of the reflection coefficient consist of the positive phase of the amplitude change and the negative phase due to a decrease in conductivity. Hooke's linear law $F=k X$ where $\mathrm{F}$ is the displacement force of the particle on the surface, $\mathrm{k}$ is the coefficient of elasticity of the metal, $X$ is the amplitude of the displacement of the particle, indicates that it is impossible to detect changes in the phase of the reflection coefficient of the microwave field for small oscillation amplitudes. The derivative of the phase $\mathrm{x}(d \varphi / d x)$ consists of a pair of components whose sign is opposite. Derivative of the amplitude fluctuations of the surface $d \varphi / d x_{S}$ opposite of the derivative of the density of the metal $d \varphi / d x_{\sigma}\left(d \varphi / d x_{S}=-\right.$ $d \varphi / d x_{\sigma}$ ). In violation of the linearity of Hooke's law (to compress the metal is always more difficult than to break) it becomes possible to detect vibrations of the metal surface. This is what happens in practice. But we should not forget that the metal is destroyed by the amplitude of the surface vibrations of more than a micron. Therefore, the practical use of the phase modulation effect to determine the small oscillations of the metal surface of the microwave sensor is impossible.

It becomes clear why researchers of small changes (amplitudes) of surface oscillations (deformations) cannot detect phase-modulated microwave oscillations from small signals, which are successfully recorded by contact sensors.

However, nature itself allows us to detect these ultra-small oscillations (1-5 nanometers) using Doppler radar. But on one condition, the fact that Russian scientists of their Institute Kurchatov-B. V. Vasiliev and L. V. lyuboshits his hypothesis, based on the virial theorem, suggested that the electron gas of metals in compression can generate an electric field that changes the surface density of conductive particles (electrons). Members of the research team, Professor V. Gorbunova I. we carried out practical tests of metal samples, measuring the level of the useful signal by Doppler radar (changes in the phase of the reflection coefficient) under double mechanical and ultrasonic loading in the presence of hidden defects. It is the latent defects that are the generators of an additional electric field at the "pressure" of ultrasound on them. Generators of the third phase component of the reflection coefficient $d \varphi / d x g(\sigma)$, which is successfully indicated by the radar Doppler frequency of $30-40 \mathrm{GHz}$. This component refers to the wave of surface conductivity. It has a velocity of propagation close to the speed of light, respectively; the wavelength is much longer than the wavelength of ultrasound.

The obtained positive results confirmed the hypothesis of B. V. Vasilyev and allowed to create a new, unusual indicator of metal defects in the future. The new device, working on a newly discovered effect (the interaction of ultrasound and conduction electrons), called the Gorbunov effect, not only allowed to overcome the seemingly overly difficult obstacle, but also to obtain new unusual results on the indexing of metal defects.

\section{Test Result}

The tests lasted for several years (1995-2012) and were accompanied by a gradual improvement in the quality, sensitivity, ease of use of the device.

The final version of the device makes it possible to detect hidden defects of metal objects with the size of a unit of microns [10] (cracks in the internal surfaces of high-pressure steam pipelines, chemical synthesizer reactors for liquid and solid polymerization of natural gas products, delamination of metal sheets $(0.3-5 \mathrm{~mm})$ made by rolling). Moreover, these defects can now be detected without additional mechanical loading. Replacing "that the external pressure is" one of the ultrasonic source is a low power $\left(10-20 \mathrm{~W} / \mathrm{cm}^{2}\right)$. If earlier defects in the rolling type of bundles could be detected by a contact sensor of 10-20 $\mathrm{MHz}$ frequency, scanning the metal surface for 510minutes, now such a marriage is indicated for 1-2 seconds without scanning the surface, since the frequency of additional ultrasound is $40-50 \mathrm{KHz}$. Two orders of magnitude lower. This property of the new device, obtained as a result of experiments, significantly increases the area of operation by reducing active losses. Scanning is excluded, defect indexing time is reduced. The depth of penetration of additional ultrasound (5-6meters) allows the use of a new device for flaw detection of large objects, length.

The working distance of the radar Doppler the package is $80-160 \mathrm{~mm}$. The radiated power of the generator built on the Gunn diode is $10 \mathrm{~mW}$. For ease of configuration the device contains AC, automatic phase control, can be independently adjusted for maximum sensitivity, the phase 
detector of the radar. Currently, the ultrasound generator, the excitation of the investigated object is a contact sensor of the type MA40MF14-5B with power amplifier frequencies of $40-50 \mathrm{KHz}$, which is developing an ultrasonic transmitter, the amplitude of the 80-90 Volt sinusoidal signal.

These properties of the new device are especially in demand for space production technology. There is a practical possibility of remote detection of riveting defects, welding, coating strength using a laser ultrasound source and Doppler radar as a sensitive sensor. All operations can be carried out in motion, during continuous tests, without stopping the process.

It should be noted that the features of the new nondestructive testing sensor are that the indication of defects of the metal object is a hollow metal vessel, involves the location of the Doppler radar outside. Experimental studies of a $300 \mathrm{~mm}$ diameter pipeline segment containing defects (cracks along the cylinder generatrix) proved that a useful signal exists only on the outer surface. The size of the pipeline allows Doppler radars to be investigated only when the length of the electromagnetic wave having an ultrasound frequency fits as a quarter of the wavelength inside the metal hollow object. So for a diameter of $300 \mathrm{~mm}$ ultrasound frequency should be $38 / 1,2=140 \mathrm{MHz}$.

Another feature of the detection of defects by the new device is that experimental studies have shown a significant dependence of the sensitivity of the entire device on the time of occurrence of defects. There is a dependence of "defect activity" on time. Some defects may lose the detection property after a few hours after the occurrence. Fortunately, this untidiness can be eliminated by heating the metal object to a temperature of $250^{\circ}-300^{\circ}$ followed by rapid cooling with water. Sensitivity is restored and continues for several hours.

The performed experiments have shown that the new nondestructive testing device is able to detect defects that are difficult to detect by traditional ultrasonic flaw detectors (it is assumed that the plane of the crystal lattice in the sample of the destroyed shaft, micro fractures, in the sample of the steam line). Butt welding defect (not detected by ultrasonic method).

In contrast to the acoustic emission method, a useful signal from the new device is obtained without the use of additional mechanical loading. For a short period of time, (2-3 seconds) ten times less than in tests by acoustic emission. In contrast to the method of ultrasound diagnostics, it was not required to use the "ideal" sample that does not contain defects.

The experiments became possible thanks to the active participation of the staff of the research laboratory of flaw detection of the Kemerovo Polytechnic University, doctor of technical Sciences Professor A. N. Smirnov, ctn., associate Professor of KSU Ababkov N. V.

The presented brief description of the device and its properties does not limit all the features of the construction, configuration and operation of the new device. I hope the appeal of interested persons to our address will allow you to resolve any issues.

\section{References}

[1] Contactless acoustic emission Converter, D. Merson, Togliatti state University, Togliatti, journal of strength physics and intelligent diagnostic systems, 2014.

[2] Gorbunov V. I., Grankin A. P. and others A. S. № 1578636. Method for recording acoustic emission signals. Priority of the invention 4364845, registered 28 October 1987.

[3] Darznek S. A., Jacobi J., Laser interferometric nanodisplacement meter, /RAS, proceedings of General physics Institute. A. M. Prokhorova, volume 62, 2006.

[4] Podymova N., Karabutov A., Belyaev I., Broadband laserultrasonic spectroscopy for quantitative evaluation of porosity effect on acoustic attenuation and phase velocity in CFRP composites/ECNDT, Prague, October 2014, p 2001.

[5] Remote acoustic emission Converter. V. I. Gorbunov, V. A. Sutorikhin,/Journal Of Applied Physics", Tomsk, 1995. - DEP. in VINITI 26. 06. 95 №1869-B 95. 4 PP.

[6] G. C. Smith and S. S. Lee, Method for detecting tool wear on a CNC lathe using a Doppler radar detector, Int. J. Adv. Manufact. Technol., vol. no. 3-4 pp. 270-280, 2004.

[7] The POSSIBILITY of INSPECTION of METAL PARTS of the microwave FIELD V. I. Gorbunov, V. A. Sutorikhin/Technical acoustics. 2010. Vol. 10. P. 16.

[8] Metod for detection acoustic emission using a microwave Dopler radar detector. /Inventor: Gregory C. Smith. /Patent No US 7. 397. 421. B2, Jul. 8, 2008.

[9] Kapranov B., Sutorikhin V., The theoretical limit on the measurement of the signals of Acoustic Emission the Doppler., NORWEGIAN JOURNAL OF DEVELOPMENT OF THE INTERNATIONAL SCIENCE 2017. P. 23-26.

[10] Remote Acoustic Emission Monitoring of Metal Ware and Welded Joints To cite this article: Boris I Kapranov and Vladimir A Sutorikhin/2017 IOP Conf. Ser.: Mater. Sci. Eng. 253012005 IOP Conference Series: Materials Science and Engineering. 